\title{
The prevalence of hyperuricemia and its correlates in Ganzi Tibetan Autonomous Prefecture, Sichuan Province, China
}

\author{
Xin Zhang ${ }^{1}$, Qingtao Meng ${ }^{1}$, Jiayue Feng ${ }^{1}$, Hang Liao ${ }^{1}$, Rufeng Shi ${ }^{1}$ Di Shi ${ }^{1}$, Lachu Renqian², Zeren Langtai \\ Yuanbin Diao ${ }^{3}$ and Xiaoping Chen ${ }^{1 *}$
}

\begin{abstract}
Background: Hyperuricemia is a common and serious public health problem. There has been no broad epidemiological survey of hyperuricemia in China, especially in Tibetan area. This study was therefore investigated the prevalence of hyperuricemia and its correlated factors among people aged 18-85 years in Ganzi Tibetan Autonomous Prefecture, Sichuan Province, China.

Methods: We carried out a cross-sectional study among 3093 participants in Ganzi Tibetan Autonomous Prefecture using questionnaires in face-to-face interviews, anthropometric measurements and biochemical tests. We included 1416 subjects with complete data including serum uric acid and medical history to analyze the prevalence of hyperuricemia and correlated factors. Hyperuricemia was defined as a fasting serum uric acid level higher than $420 \mu \mathrm{mol} / \mathrm{L}$ in men and $360 \mathrm{\mu mol} / \mathrm{L}$ in women.

Results: The overall crude prevalence of hyperuricemia was 37.2\%, and was greater in men than women (41\% vs 34.4\%, $P=0.011$ ). The age-adjusted prevalence was 33.0\%. Characteristics linked to hyperuricemia were farmers-herdsmen (OR: 1.749, 95\% Cl: 1.022-2.992), low to moderate education level (low OR:1.57, 95\% Cl: 1.102-2.237; moderate OR: 1.86, 95\% Cl: 1.167-2.963), current drinking (OR: 1.795, 95\% Cl: 1.193-2.702), hypertension (OR: 1.48, 95\% Cl: 1.091-2.006), higher body mass index (1 unit increase) (OR: 1.116, 95\% Cl: 1.077-1.156) and higher serum creatinine (1 unit increase) (OR: 1.046, 95\% CI: 1.034-1.059). Serum uric acid was positively related to triglycerides and total cholesterol and negatively related to high density lipoprotein cholesterol in all subjects. Hyperuricemia was a risk factor for high triglyceride ((OR: 2.13, 95\% Cl: 1.156-3.9266) and high total cholesterol (OR: 2.313, 95\% Cl: 1.364-3.923) in men and for high low-density lipoprotein cholesterol (OR: 2.696, 95\% Cl: 1.386-5.245) in women.
\end{abstract}

Conclusion: There is a high prevalence of hyperuricemia in Ganzi Tibetan Autonomous Prefecture. The government needs to prevent and manage hyperuricemia in this area.

Keywords: Hyperuricemia, Uric acid, Prevalence, Tibetan, Tibet, Risk

\section{Background}

Hyperuricemia is defined as a fasting serum uric acid higher than $420 \mu \mathrm{mol} / \mathrm{L}$ in men and $360 \mu \mathrm{mol} / \mathrm{L}$ in women [1]. It causes gout and urinary calculi and is also related to a variety of comorbidities, including cardiovascular diseases, chronic kidney disease, metabolic syndrome, diabetes mellitus [2]. Previous studies have shown

\footnotetext{
* Correspondence: xiaopingchen111@126.com

${ }^{1}$ Department of Cardiology, West China Hospital, Sichuan University,

Chengdu, Sichuan 610000, People's Republic of China

Full list of author information is available at the end of the article
}

that serum uric acid is affected by age, sex, ethnicity, dietary habits, environment and other factors [1].

The United States National Health and Nutrition Examination Survey 2007-2008 study showed that the prevalence of Hyperuricemia was $21.2 \%$ among men and 21.6\% among women in America [3]. The China National Survey of Chronic Kidney Disease showed that the age-adjusted prevalence of hyperuricemia among Chinese adults in 2009-2010 was 8.4\% (9.9\% in men and $7.0 \%$ in women) [4]. A recent meta-analysis of 36 articles from 2000 to 2014 showed that the prevalence of

(C) The Author(s). 2018 Open Access This article is distributed under the terms of the Creative Commons Attribution 4.0 International License (http://creativecommons.org/licenses/by/4.0/), which permits unrestricted use, distribution, and 
hyperuricemia and gout in Mainland China was 13.3\% [5]. Socioeconomic developments and lifestyles changes in China mean that the prevalence of hyperuricemia has increased in the last few decades, with a trend toward onset at younger ages. A previous investigation in Mainland China showed that the condition is more prevalent in men than women, in cities than rural areas, and in coastal than inland areas [1, 4]. However there have been few broad epidemiological surveys of hyperuricemia in China, especially in remote and underdeveloped areas.

Ganzi Tibetan Autonomous Prefecture is a large high altitude area of Tibet, with distinct geographic characteristics and lifestyles compared with other areas of mainland China. People tend to eat a high-salt, fat-rich diet and drink alcohol, so the cardiovascular risk factors and level of cardiovascular disease are more serious [6-8]. Most studies in this area have investigated the prevalence of cardiovascular disease and risk factors including hypertension, diabetes mellitus, overweight or obesity, and dyslipidemia in Tibetan area $[6,9,10]$. Few studies have focused on determining the prevalence and epidemiological features of hyperuricemia in this region.

It is important to obtain more epidemiological data on chronic disease and cardiovascular risk factors to improve medical and health status this area. This study therefore examined the prevalence of hyperuricemia and its correlated factors among people aged 18-85 years in Ganzi Tibetan Autonomous Prefecture, Sichuan Province, China founded by Sichuan science and technology department.

\section{Methods}

\section{Study subjects}

Ganzi Tibetan Autonomous Prefecture is located in western Sichuan province, China. From September 2016 to November 2017, a representative sample of participants aged 18 years and over was selected to investigate the prevalence and risk factors of chronic cardiovascular disease including hypertension, dyslipidemia, diabetes mellitus and hyperuricemia. This investigation used a multi-stage, stratified, random-cluster sampling scheme. Two counties in the prefecture (Jiulong and Seda) were selected first. In the second stage, a town was randomly selected in Jiulong county, and a town and a Buddhist college in Seda county. All the eligible participants 18 years and over were included. We excluded pregnant women, and those with malignant tumors, mental health problems or having artificial extracorporeal liver support and hemodialysis or peritoneal dialysis as a result of severe hepatic or kidney failure. In total, We included 1416 subjects with information about serum uric acid and medical history out of 3093 subjects overall. This study was approved by the Ethics committee of West China Hospital, Sichuan University (Chengdu, China).
All of the participants gave informed consent after had been given information about the objectives and benefits of our study.

\section{Survey method and data collection}

Data were collected by investigators during face-to-face interviews using a standardized questionnaire. All investigators received training in the objective of the study, the method of measurement, the significance of standardization and how to complete the questionnaire.

Blood pressure readings were taken from participants' right arms at a time between 8:00 a.m. and 12:00 noon using a standardized automatic electronic sphygmomanometer (Omron HEM-770A). Readings were taken three times at 2-min intervals after at least 5 min of rest in a warm and quiet indoor place [11]. Systolic blood pressure (SBP) and diastolic blood pressure (DBP) were taken as the mean of three readings.

Weight and height were measured to the nearest $0.2 \mathrm{~kg}$ and $1 \mathrm{~cm}$. Waist circumference (WC) was measured at the umbilicus using a non-elastic tape (to the nearest $0.5 \mathrm{~cm}$ ) at the level midway between the lateral rib margin and the iliac crest in the end of a normal exhalation. Hip circumference $(\mathrm{HC})$ was measured at the level of the greater trochanter [12]. Body mass index (BMI) was calculated as the weight in kilograms divided by the square root of the height in meters. Waist-to-hip ratio (WHR) was calculated as WC divided by HC.

\section{Laboratory measurements}

Blood samples were obtained in the morning after overnight fasting. Biochemistry parameters were measured at the laboratory of West China Hospital (Chengdu, China). Total cholesterol (TC), triglycerides (TG), and fasting plasma glucose (FPG) were all determined using the enzymatic method, and high-density lipoprotein cholesterol (HDL-C) was measured using the phosphotungstic acid/magnesium chloride precipitation method. The concentration of low-density lipoprotein cholesterol (LDL-C) was calculated using the Friedewald formula. Creatinine was tested by picric acid Jaffe method and uric acid by kinetic UV assay [13].

\section{Definitions}

Hyperuricemia was defined as a fasting serum uric acid higher than $420 \mu \mathrm{mol} / \mathrm{L}$ in men and $360 \mu \mathrm{mol} / \mathrm{L}$ in women, in line with Chinese guideline [6]. Hypertension was defined as a SBP of at least $140 \mathrm{mmHg}$, and/or a DBP of at least $90 \mathrm{mmHg}$, and/or use of antihypertensive medication. Dyslipidemia was defined using the National Cholesterol Education Program-Third Adult Treatment Panel (ATP III) criteria [14] . High TC was defined as TC of at least $6.21 \mathrm{mmol} / \mathrm{L}(240 \mathrm{mg} / \mathrm{dL})$. Low HDL-C was defined as less than $1.03 \mathrm{mmol} / \mathrm{L}(40 \mathrm{mg} / \mathrm{dL})$. High LDL-C was de 
fined as at least $4.16 \mathrm{mmol} / \mathrm{L}(160 \mathrm{mg} / \mathrm{dL})$. High TG was defined as at least $2.26 \mathrm{mmol} / \mathrm{L}(200 \mathrm{mg} / \mathrm{dL})$. Diabetes mellitus was diagnosed using WHO criteria: FPG of at least $7 \mathrm{mmol} / \mathrm{L}(126 \mathrm{mg} / \mathrm{dL})$ and/or being treated for diabetes [15].

Current smokers were defined as smoking at least one cigarette per day during the last year [16]. Current drinkers were defined as drinking alcohol at least 12 times during the last year [17]. A vegetarian diet was defined as one that did not contain meat, poultry or fish but did contain eggs and dairy, plus plant-based foods, such as fruits, vegetables, whole grains, legumes, nuts and seeds. A nonvegetarian diet contained meat, poultry or fish, vegetables and fruits [18]. Participants were also asked whether they drank buttered tea for breakfast. People at high altitude often take only mild to moderate intensity activity because of the hypoxic environment. We classified physical activity per day by exercise time into four levels: less than $30 \mathrm{~min}$ as level 1, $30 \mathrm{~min}-1 \mathrm{~h}$ as level 2, 1-1.5 h as level 3, and more than $1.5 \mathrm{~h}$ as level 4 . Education level was assessed as illiteracy, primary school, middle school, or high school and higher.

\section{Statistics}

All the statistical analyses used SPSS version 23.0. Descriptive statistics were calculated for all the variables. Continuous variables were reported as mean values and standard deviations and categorical variables as numbers and percentages. The baseline data were evaluated using Student's $t$ test, ANOVA or the $\chi^{2}$ test, depending on data types. Simple correlation analysis and multiple linear regression were used to analyze the relationship between serum uric acid and the index of cardiometabolic risk factors. Multivariate logistic regression analyses were used to identify factors influencing hyperuricemia. Odds ratios (ORs) with 95\% confidence intervals (CIs) were used to quantify the relationship. $P$ values less than 0.05 were considered to be statistically significant.

\section{Results}

\section{Baseline characteristics of study population}

Table 1 shows the baseline characteristics. Current smoking and drinking were more frequent in men than women, but women were more likely to drink buttered tea and eat meat. Height, weight, heart rate, serum uric acid, glucose and serum creatinine were significantly greater for men than women. Age, serum cholesterol, serum HDL cholesterol and glucose were significantly greater in women than men, with no difference in BMI, WC, HC, WHR, SBP, DBP, serum LDL-C and serum TG between the two genders. The percentage of monks/ nuns, farmers-herdsmen and ordinary residents were $37.8 \%, 25 \%$ and $37.2 \%$ for men and $26.3 \%, 41 \%$ and $32.3 \%$ for women. There were no differences in ethnic composition, education level, physical activity level and prevalence of primary hypertension.

\section{Prevalence of hyperuricemia in different genders and age groups}

The overall crude prevalence of hyperuricemia was $37.2 \%$. It was more prevalent in men than women $(41 \%$ vs $34.4 \%, P=0.011$ ), and the serum uric acid level was also higher for men than women overall (408.45 \pm 105.35 vs $333.27 \pm 84.73 \mu \mathrm{mol} / \mathrm{L}, P<0.001)$ and in age cohorts. The prevalence of hyperuricemia and average serum uric acid in different age groups was shown in Fig. 1. The age-adjusted prevalence of hyperuricemia was $33.0 \%$ using the most recent population statistics for Ganzi Tibetan Autonomous Prefecture.

\section{Correlated factors and hyperuricemia}

Multivariable logistic regression showed that being farmers-herdsmen ((OR: 1.749, 95\% CI: 1.022-2.992), having low to moderate education level ((low OR: 1.57, 95\% CI: 1.102-2.237; moderate OR: 1.86, 95\% CI: $1.167-$ 2.963), current drinking OR: 1.795, 95\% CI: $1.193-$ 2.702), hypertension ((OR: 1.48, 95\% CI: 1.091-2.006), higher BMI (1 unit increase) (OR: 1.116, 95\% CI: 1.0771.156) and higher creatinie (1 unit increase) (OR: 1.046, 95\% CI: 1.034-1.059) were related to hyperuricemia in all subjects (Table 2). For men, low education level, current drinking, higher BMI and higher serum creatinine were risk factors for hyperuricemia. Being Tibetan compared to Han and doing high levels of physical activity were protective factors for hyperuricemia in male group. For women, the multivariable logistic regression showed that being farmers-herdsmen and residents rather than nuns, moderate education level, current drinking, hypertension, higher BMI and higher serum creatinine were risk factors for hyperuricemia (Table 3).

\section{Hyperuricemia and cardiometabolic index}

Simple correlation analysis (Table 4) showed that serum uric acid was positively related to TG, TC, and LDL-C in all subjects, and for men women separately. It was negatively related to HDL-c in all subjects and women alone, but not men alone. Multiple linear regression (Table 5) showed that serum uric acid was positively related to TG and TC and negatively related to HDL-c in all subjects after adjusting for age, sex, BMI, serum creatinine, smoking, drinking alcohol, hypertension, chronic kidney disease, and diabetes mellitus. In the overall group, the prevalence of hyperuricemia was higher in subjects with high TG, high TC or high LDL-c, and the average serum uric acid was higher in subjects with high TG, high TC, high LDL-c or low HDL-c (Fig. 2). After adjusting confounding factors including age, BMI, WHR, ethnicity, occupation, education level, drinking buttered tea, eating 
Table 1 Demographic, anthropometric, and plasma biochemical characteristics of subjects in Ganzi Tibetan Autonomous Prefecture, Sichuan Province, China

\begin{tabular}{|c|c|c|c|c|}
\hline Characteristics & $\begin{array}{l}\text { Male } \\
(N=614)\end{array}$ & $\begin{array}{l}\text { Female } \\
(N=802)\end{array}$ & $\begin{array}{l}\text { Hyperuricemia } \\
(N=528)\end{array}$ & $\begin{array}{l}\text { Non-hyperuricemia } \\
(N=888)\end{array}$ \\
\hline \multicolumn{5}{|l|}{ Clinic variables } \\
\hline Age (years) & $50.31 \pm 16.84$ & $52.21 \pm 13.94$ \# & $54.98 \pm 13.56$ & $49.37 \pm 15.81^{* *}$ \\
\hline Height & $165.19 \pm 7.02$ & $156.38 \pm 6.72$ \#\#: & $160.85 \pm 8.57$ & $159.81 \pm 7.82 *$ \\
\hline Weight & $69.55 \pm 12.25$ & $62.97 \pm 11.39^{\# \#}$ & $69.19 \pm 12.76$ & $63.77 \pm 11.41^{* *}$ \\
\hline BMl & $25.45 \pm 4.01$ & $25.73 \pm 4.29$ & $26.69 \pm 4.22$ & $24.95 \pm 4.00^{* *}$ \\
\hline WC & $85.64 \pm 10.17$ & $85.57 \pm 11.90$ & $89.28 \pm 9.53$ & $83.36 \pm 11.53^{* *}$ \\
\hline $\mathrm{HC}$ & $94.56 \pm 8.34$ & $94.45 \pm 0.08$ & $96.17 \pm 8.22$ & $93.48 \pm 8.11^{* *}$ \\
\hline WHR & $0.91 \pm 0.84$ & $0.91 \pm 0.10$ & $0.93 \pm 0.07$ & $0.89 \pm 0.11^{* *}$ \\
\hline SBP & $134.75 \pm 24.19$ & $134.87 \pm 25.51$ & $140.06 \pm 24.36$ & $131.86 \pm 24.78^{* *}$ \\
\hline DBP & $82.44 \pm 15.79$ & $81.38 \pm 14.51$ & $86.05 \pm 14.51$ & $79.48 \pm 14.84^{* *}$ \\
\hline$H R$ & $81.4 \pm 14.22$ & $79.39 \pm 13.93$ \# & $79.43 \pm 13.86$ & $80.74 \pm 14.20$ \\
\hline \multicolumn{5}{|l|}{ Biochemical variables } \\
\hline TC & $5.03 \pm 1.30$ & $5.30 \pm 1.21$ \#\#: & $5.58 \pm 1.19$ & $4.95 \pm 1.23^{* *}$ \\
\hline LDL -c & $2.83 \pm 0.92$ & $2.89 \pm 0.87$ & $3.13 \pm 0.90$ & $2.71 \pm 0.85^{* *}$ \\
\hline $\mathrm{HDL}-\mathrm{C}$ & $1.29 \pm 0.37$ & $1.48 \pm 0.38^{\# \#:}$ & $1.38 \pm 0.39$ & $1.41 \pm 0.38$ \\
\hline TG & $1.47 \pm 1.09$ & $1.44 \pm 1.17$ & $1.76 \pm 1.27$ & $1.28 \pm 1.01^{* *}$ \\
\hline UA & $408.5 \pm 105.4$ & $333.27 \pm 84.73^{\# \#:}$ & $462.17 \pm 79.88$ & $308.50 \pm 61.28^{* *}$ \\
\hline Creatinine & $84.18 \pm 15.77$ & $71.97 \pm 12.71$ \#\#: & $83.68 \pm 16.47$ & $73.43 \pm 13.25^{* *}$ \\
\hline Glucose & $5.13 \pm 1.62$ & $5.34 \pm 1.37^{\#}$ & $5.38 \pm 1.49$ & $5.18 \pm 1.48 *$ \\
\hline \multicolumn{5}{|l|}{ Lifestyle variables } \\
\hline Drinking buttered tea & $44 \%$ & $57.8 \%$ \#\#: & $64 \%$ & $44.4 \%{ }^{* *}$ \\
\hline Tea with salt & $47.20 \%$ & $61.7 \%$ \#\#: & $65.10 \%$ & $49.6 \%{ }^{* *}$ \\
\hline Current smoking & $31.10 \%$ & $2.8 \%$ \#\#: & $22 \%$ & $10.8 \%{ }^{* *}$ \\
\hline Currrent drinking & $20 \%$ & $7.4 \%$ \#\#: & $20.40 \%$ & $8.2 \%^{* *}$ \\
\hline Eating meat & $76.80 \%$ & $84 \%$ \#\#: & $91.50 \%$ & $74.4 \%{ }^{* *}$ \\
\hline Physical activity & 0.067 & & $<0.001$ & \\
\hline Level 1 & $11.20 \%$ & $13.60 \%$ & $15.40 \%$ & $10.90 \%$ \\
\hline Level 2 & $17.80 \%$ & $17 \%$ & $21.90 \%$ & $15.00 \%$ \\
\hline Level 3 & $11 \%$ & $15.00 \%$ & $17.50 \%$ & $10.90 \%$ \\
\hline Level 4 & $59.80 \%$ & $54.10 \%$ & $45.20 \%$ & $63.10 \%$ \\
\hline \multicolumn{5}{|l|}{ Else variables } \\
\hline HTN & $49.20 \%$ & $48.60 \%$ & $63.50 \%$ & $40.4 \%{ }^{* *}$ \\
\hline DM & $5.40 \%$ & $6.20 \%$ & $7.10 \%$ & $5.10 \%$ \\
\hline CKD & $3.30 \%$ & $2.50 \%$ & $5.50 \%$ & $0.8 \% *$ \\
\hline Allopurinol & $0 \%$ & $0 \%$ & $0 \%$ & $0 \%$ \\
\hline Benzbromarone & $0 \%$ & $0 \%$ & $0 \%$ & $0 \%$ \\
\hline Hydrochlorothiazide & $0.81 \%$ & $0.99 \%$ & $0.94 \%$ & $0.91 \%$ \\
\hline Education & 0.138 & & 0.001 & \\
\hline 1 & $53.60 \%$ & $59.30 \%$ & $46.60 \%$ & $65.10 \%$ \\
\hline 2 & $19.10 \%$ & $18.30 \%$ & $23.70 \%$ & $15.80 \%$ \\
\hline 3 & $13.90 \%$ & $11.80 \%$ & $19.50 \%$ & $8.70 \%$ \\
\hline 4 & $13.40 \%$ & $10.60 \%$ & $14.10 \%$ & $10.40 \%$ \\
\hline
\end{tabular}


Table 1 Demographic, anthropometric, and plasma biochemical characteristics of subjects in Ganzi Tibetan Autonomous Prefecture, Sichuan Province, China (Continued)

\begin{tabular}{|c|c|c|c|c|}
\hline Characteristics & $\begin{array}{l}\text { Male } \\
(N=614)\end{array}$ & $\begin{array}{l}\text { Female } \\
(N=802)\end{array}$ & $\begin{array}{l}\text { Hyperuricemia } \\
(N=528)\end{array}$ & $\begin{array}{l}\text { Non-hyperuricemia } \\
(N=888)\end{array}$ \\
\hline Ethnicity & 0.572 & & $<0.001$ & \\
\hline Han & $20.90 \%$ & $20.80 \%$ & $28.40 \%$ & $16.40 \%$ \\
\hline Tibetan & $69.50 \%$ & $71.10 \%$ & $58.80 \%$ & $77.10 \%$ \\
\hline Yi & $9.70 \%$ & $8.10 \%$ & $12.80 \%$ & $6.50 \%$ \\
\hline Occupation & $<0.001$ & & $<0.001$ & \\
\hline Monks & $37.80 \%$ & $26.30 \%$ & $15.60 \%$ & $40.60 \%$ \\
\hline Farmers-herdsmen & $25 \%$ & $41 \%$ & $39.10 \%$ & $31.50 \%$ \\
\hline Residents & $37.20 \%$ & $32.30 \%$ & $45.30 \%$ & $27.90 \%$ \\
\hline
\end{tabular}

$B M I$ body mass index, WC waist circumference, HC hip circumference, WHR Waist-to-hip ratio, SBP systolic blood pressure, DBP diastolic blood pressure, TC Total cholesterol, TG triglycerides, $L D L-c$ low-density lipoprotein cholesterol, HDL-C high-density lipoprotein cholesterol, UA uric acid, DM diabetes mellitus, CKD chronic kidney disease; Education level was assessed as illiteracy, primary school, middle school, or high school and higher for 1,2,3,4; Physical activity was classified by exercise time into 4 levels, less than 30 min level 1, 30 min-1 h level 2,1-1.5 h level 3, more than $1.5 \mathrm{~h}$ level 4 . ${ }^{\#}: P<0.05$; ${ }^{\# \#}: P<0.001$, male Versus female; ${ }^{*}: P<0.05$; **: $P<0.001$, Hyperuricemia group Versus Non-hyperuricemia group

meat, physical activity, and current smoking and drinking, the multivariable logistic regression showed that hyperuricemia was a risk factor for high TG (OR: 2.13, 95\% CI: 1.156-3.926) and high TC (OR: 2.313, 95\% CI: 1.364-3.923) for men and for high LDL-c (OR: 2.696, 95\% CI: 1.386-5.245) for women respectively (Table 6).

\section{Discussion}

This study found that the prevalence of hyperuricemia in this area of Tibet was significantly higher than other

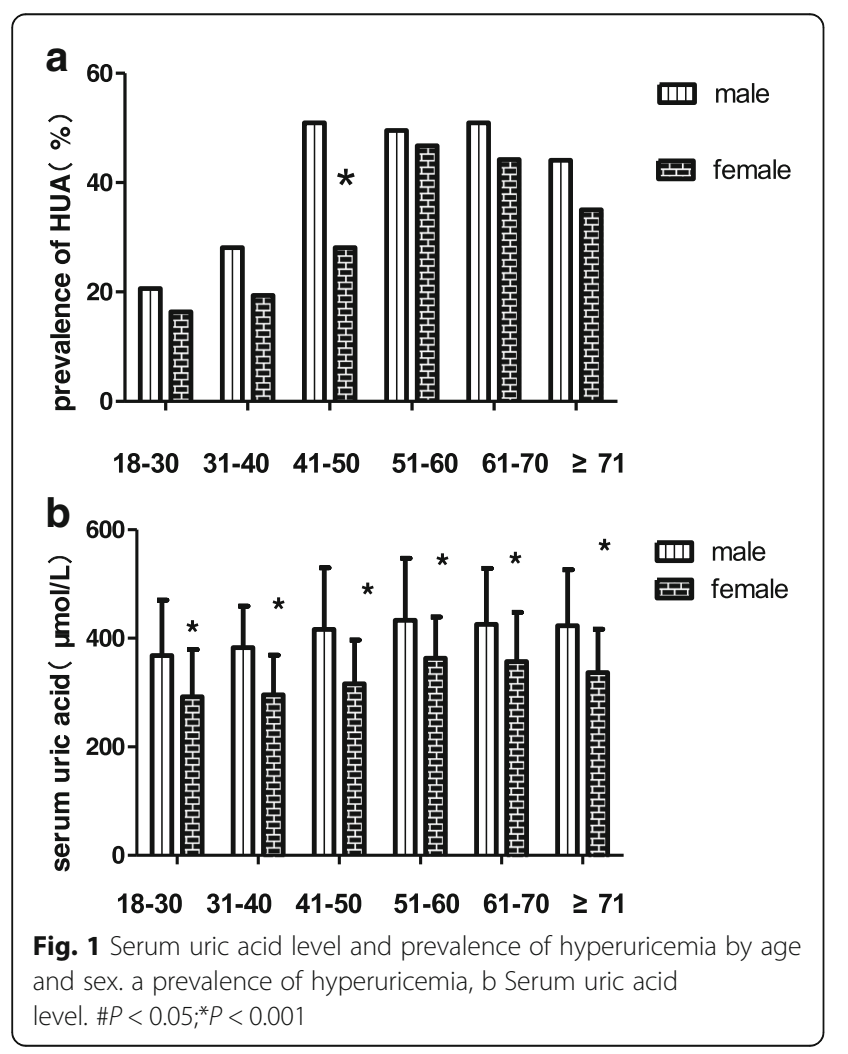

areas of Mainland China. The lifestyle in this area differs from that in inland and coastal areas of China. Firstly, in high altitude areas, food resources are limited and food diversity is insufficient when measured using the 11-item Food Diversity Score Kyoto (FDSK-11) [19]. A national survey on dietary pattern and meal behavior in China demonstrated that Tibetan ate lower legumes than other groups including Yi, Han, and Zhuang [20]. Secondly, people in Tibetan area liked eating traditional salty yak buttermilk tea, mushrooms and yak meat, which are specialties of the plateau region. Finally, drinking is a popular way to entertain guests and keeping warm $\mathrm{fn}$ the area, because of both the alpine climate and traditional customs. A survey of alcohol drinking behavior among native adult Tibetans in Lhasa demonstrated that the overall alcohol drinking rate was $48.95 \%$ (64. $16 \%$ for men and $36.00 \%$ for women), which is higher than the national average [21]. In other words, people in Tibet had a distinct dietary structure, giving priority to meat, alcohol, Fat-rich diet. Certain food, including meat, alcohol and mushrooms can increase the risks of hyperuricemia [21-23].

We found that current drinking, hypertension, higher BMI and creatinine, being farmers-herdsmen, and having low to moderate education level were risk factors for hyperuricemia. Alcohol may increase uric acid by increasing in lactic acid exchange with urate in kidneys via sodium-dependent monocarboxylate transporters SLC5A8 and SLC5A12 upregulation of urate transporter 1 (URAT1) [24]. Alcohol has also been shown to increase uric acid production by increasing adenosine triphosphate degradation to adenosine monophosphate [25]. One prospective study in Japan, confirmed that habitual alcohol intake contributed to the development of hyperuricemia, regardless of type of alcohol consumed [26]. Our study confirmed the positive association 
Table 2 Multiple regression analyses of hyperuricemia and associated factors in all subjects

\begin{tabular}{|c|c|c|c|c|c|}
\hline & \\
\hline Variables & OR & $95 \% \mathrm{Cl}$ & Variables & Male & Female \\
\hline Female & 0.903 & $(0.629-1.297)$ & & OR $(95 \% \mathrm{Cl})$ & OR $(95 \% \mathrm{Cl})$ \\
\hline Age(years) & & & Age(years) & & \\
\hline $18-30$ & 1 & (reference) & $18-30$ & 1.00 (reference) & 1.00 (reference) \\
\hline $31-40$ & 0.618 & $(0.326-1.17)$ & $31-40$ & $0.673(0.297-1.521)$ & $0.459(0.146-1.44)$ \\
\hline $41-50$ & 0.818 & $(0.442-1.515)$ & $41-50$ & $1.289(0.56-2.965)$ & $0.469(0.159-1.388)$ \\
\hline $51-60$ & 0.836 & $(0.431-1.622)$ & $51-60$ & $0.721(0.277-1.878)$ & $0.644(0.211-1.969)$ \\
\hline $61-70$ & 0.893 & $(0.459-1.735)$ & $61-70$ & $0.793(0.311-2.022)$ & $0.72(0.23-2.261)$ \\
\hline$\geq 71$ & 0.601 & $(0.289-1.251)$ & $\geq 71$ & $0.515(0.185-1.434)$ & $0.55(0.16-1.887)$ \\
\hline Ethnicity & & & Ethnicity & & \\
\hline Han & 1 & (reference) & Han & 1.00(reference) & 1.00(reference) \\
\hline Tibetan & 0.71 & $(0.501-1.006)$ & Tibetan & $0.502(0.284-0.885)$ & $0.885(0.557-1.407)$ \\
\hline$Y_{i}$ & 1.054 & $(0.653-1.701)$ & Yi & $1.443(0.681-3.06)$ & $0.929(0.47-1.838)$ \\
\hline Occupation & & & Occupation & & \\
\hline Morks & 1 & (reference) & Morks & 1.00(reference) & 1.00(reference) \\
\hline Farmers-herdsmen & 1.749 & $(1.022-2.992)$ & Farmers-herdsmen & $1.321(0.579-3.013)$ & $2.78(1.282-6.027)$ \\
\hline Residents & 1.697 & $(0.905-3.183)$ & Residents & $1.1(0.404-2.994)$ & $2.544(1.055-6.133)$ \\
\hline Education & & & Education & & \\
\hline 1 & 1 & (reference) & 1 & 1.00(reference) & 1.00(reference) \\
\hline 2 & 1.57 & $(1.102-2.237)$ & 2 & $1.774(1.012-3.11)$ & $1.371(0.849-2.215)$ \\
\hline 3 & 1.86 & $(1.167-2.963)$ & 3 & $1.296(0.619-2.715)$ & $3.102(1.639-5.871)$ \\
\hline 4 & 1.195 & $(0.691-2.066)$ & 4 & $1.017(0.437-2.37)$ & $1.345(0.608-2.974)$ \\
\hline Drinking buttered tea & 0.945 & $(0.669-1.335)$ & Drinking buttered tea & $1.199(0.709-2.028)$ & $0.727(0.445-01.19)$ \\
\hline Eating meat & 1.364 & $(0.835-2.227)$ & Eating meat & $1.439(0.744-2.78)$ & $1.352(0.626-2.92)$ \\
\hline Physical activity & & & Physical activity & & \\
\hline Level 1 & 1 & (reference) & Level 1 & 1.00(reference) & 1.00(reference) \\
\hline Level 2 & 0.929 & $(0.589-1.464)$ & Level 2 & $0.735(0.353-1.53)$ & $1.243(0.669-2.311)$ \\
\hline Level 3 & 0.895 & $(0.554-1.448)$ & Level 3 & $1.01(0.446-2.29)$ & $0.962(0.505-1.829)$ \\
\hline Level 4 & 0.872 & $(0.578-1.317)$ & Level 4 & $0.468(0.24-0.911)$ & $1.546(0.885-2.702)$ \\
\hline Current smoking & 0.99 & $(0.647-1.513)$ & Current smoking & $0.809(0.483-1.357)$ & $1.652(0.591-4.616)$ \\
\hline Current drinking & 1.795 & $(1.193-2.702)$ & Current drinking & $1.1(1.014-3.14)$ & $2.224(1.156-4.278)$ \\
\hline Hypertension & 1.48 & $(1.091-2.006)$ & Hypertension & $1.33(0.822-2.154)$ & $1.549(1.026-2.338)$ \\
\hline $\mathrm{BMl}$ & 1.116 & $(1.077-1.156)$ & $\mathrm{BMI}$ & $1.1(1.04-1.164)$ & $1.123(1.07-1.178)$ \\
\hline WHR & 1.336 & $(0.299-5.959)$ & WHR & $0.833(0.054-12.76)$ & $1.522(0.241-9.626)$ \\
\hline CREA & 1.046 & $(1.034-1.059)$ & CREA & $1.034(1.019-1.05)$ & $1.075(1.05-1.1)$ \\
\hline
\end{tabular}

$B M I$ body mass index, WHR Waist-to-hip ratio, CREA creatinine Education level was assessed as illiteracy, primary school, middle school, or high school and higher for1,2,3,4; Physical activity was classified by exercise time into 4 levels, less than 30 min level 1, 30 min-1 h level 2, 1-1.5 h level 3, more than $1.5 \mathrm{~h}$ level 4. Education level was assessed as illiteracy, primary school, middle school, or high school and higher; OR odds ratio, $95 \% \mathrm{Cl}$ : $95 \%$ confidence interval. ${ }^{*} P<0.05$ for the independent association between hyperuricemia and each factor after adjusting for the remaining factors

of hypertension and hyperuricemia. The prevalence of hypertension and hyperuricemia has increased worldwide in recent years. Hypertension and HUA were both related to age, metabolism and lifestyle. BMI has
Table 3 Multiple regression analyses of hyperuricemia and associated factors in different genders

Ethnicity

1.00 (reference) $\quad 1.00$ (reference)

$1.321(0.579-3.013) \quad 2.78(1.282-6.027)$

Residents

$B M I$ body mass index, WHR Waist-to-hip ratio, CREA creatinine Education level was assessed as illiteracy, primary school, middle school, or high school and higher for1,2,3,4; Physical activity was classified by exercise time into 4 levels, less than 30 min level 1, 30 min-1 $\mathrm{h}$ level 2, 1-1.5 h level 3, more than $1.5 \mathrm{~h}$ level 4. Education level was assessed as illiteracy, primary school, middle school, or high school and higher; OR: odds ratio, $95 \% \mathrm{Cl}$ : $95 \%$ confidence interval. ${ }^{*} P<0.05$ for the independent association between hyperuricemia and each factor after adjusting for the remaining factors

been used as a surrogate variable for evaluating overweight and obesity. Choi et al. confirmed that obesity was a strong risk factor for the development of hyperuricemia and gout in men [27]. A systematic 
Table 4 Cardiometabolic risk factors associated with serum uric acid levels in simple correlation

\begin{tabular}{|c|c|c|c|c|c|c|}
\hline \multirow[t]{2}{*}{ Variables } & \multicolumn{2}{|l|}{ ALL } & \multicolumn{2}{|l|}{ Male } & \multicolumn{2}{|l|}{ Female } \\
\hline & $r$ & $P$ & $r$ & $P$ & $r$ & $P$ \\
\hline$\overline{\mathrm{TG}}$ & 0.243 & $<0.001$ & 0.289 & $<0.001$ & 0.23 & $<0.001$ \\
\hline $\mathrm{TC}$ & 0.247 & $<0.001$ & 0.37 & $<0.001$ & 0.247 & $<0.001$ \\
\hline $\mathrm{HDL}-\mathrm{C}$ & -0.12 & $<0.001$ & 0.038 & 0.346 & -0.101 & 0.004 \\
\hline LDL-C & 0.242 & $<0.001$ & 0.331 & $<0.001$ & 0.223 & $<0.001$ \\
\hline glucose & 0.03 & 0.308 & 0.046 & 0.249 & 0.074 & 0.057 \\
\hline
\end{tabular}

TG triglycerides, TC Total cholesterol, HDL-C, high-density lipoprotein cholesterol, LDL-c low-density lipoprotein cholesterol, r: correlation coefficient; P:P value

review and meta-analysis of ten prospective studies confirmed that higher BMI increases the risk of gout [28]. We also found serum creatinine level was associated with hyperuricemia and the average level of serum creatinine was higher in subjects with hyperuricemia, with a higher average level of serum creatinine in those with hyperuricemia. This result was in lines with a study in the Jinan area of China [29].

Our findings also suggest that being farmers-herdsman was a risk factor for hyperuricemia in the overall group of participants. In our study, Monks/nuns had lower levels of smoking and drinking alcohol and were more likely to be vegetarian than other groups. Smoking and drinking alcohol were risk factor of hyperuricemia. The monks/nuns reported a diet of plant-based foods, such as fruits, vegetables, whole grains, legumes, nuts and seeds and eggs, and dairy. Yi-Tsen Tsai found Chinese adults in Taiwan who ate more vegetables and fruit were more likely to have a lower uric acid concentration [30]. Szeto et al. found that a long-term vegetarian diet was

Table 5 Cardiometabolic risk factors associated with serum uric acid levels in multiple linear regression

\begin{tabular}{|c|c|c|c|c|c|c|c|}
\hline \multirow[t]{2}{*}{ Varibles } & & \multicolumn{2}{|l|}{ ALL } & \multicolumn{2}{|l|}{ Male } & \multicolumn{2}{|l|}{ female } \\
\hline & & $\beta$-value & $P$ & $\beta$-value & $P$ & $\beta$-value & $P$ \\
\hline \multirow[t]{2}{*}{$\overline{\mathrm{TG}}$} & Model 1 & 0.078 & 0.026 & 0.183 & $<0.001$ & 0.07 & 0.149 \\
\hline & Model 2 & 0.063 & 0.046 & 0.098 & 0.059 & 0.049 & 0.289 \\
\hline \multirow[t]{2}{*}{ TC } & Model 1 & 0.493 & $<0.001$ & 0.548 & $<0.001$ & 0.472 & $<0.001$ \\
\hline & Model 2 & 0.189 & 0.009 & 0.509 & 0.001 & 0.077 & 0.438 \\
\hline \multirow[t]{2}{*}{$\mathrm{HDL}-\mathrm{C}$} & Model 1 & -0.256 & $<0.001$ & -0.092 & 0.119 & -0.216 & $<0.001$ \\
\hline & Model 2 & -0.126 & 0.001 & -0.115 & 0.5 & -0.159 & 0.004 \\
\hline \multirow[t]{2}{*}{ LDL-C } & Model 1 & -0.154 & 0.023 & -0.145 & 0.165 & -0.191 & 0.029 \\
\hline & Model 2 & -0.021 & 0.722 & -0.098 & 0.338 & -0.006 & 0.937 \\
\hline \multirow[t]{2}{*}{ Glucose } & Model 1 & -0.06 & 0.034 & -0.097 & 0.019 & 0.006 & 0.878 \\
\hline & Model 2 & -0.066 & 0.7 & -0.083 & 0.49 & -0.978 & 0.329 \\
\hline
\end{tabular}

TG triglycerides, TC Total cholesterol, HDL-C high-density lipoprotein cholesterol, $L D L$-c low-density lipoprotein cholesterol; Model 1:unadjusted; Model 2: adjusted for age, sex (male $=1$, female $=2$ ), body mass index, serum creatinine, smoking (yes $=1$, no $=0$ ), drinking alcohol (yes $=1$, no $=0$ ), hypertension (yes $=1$, no $=0$ ), Chronic kidney disease (yes $=1$, no $=0$ ), diabetes mellitus (yes $=1$, no $=0), P: P$ value

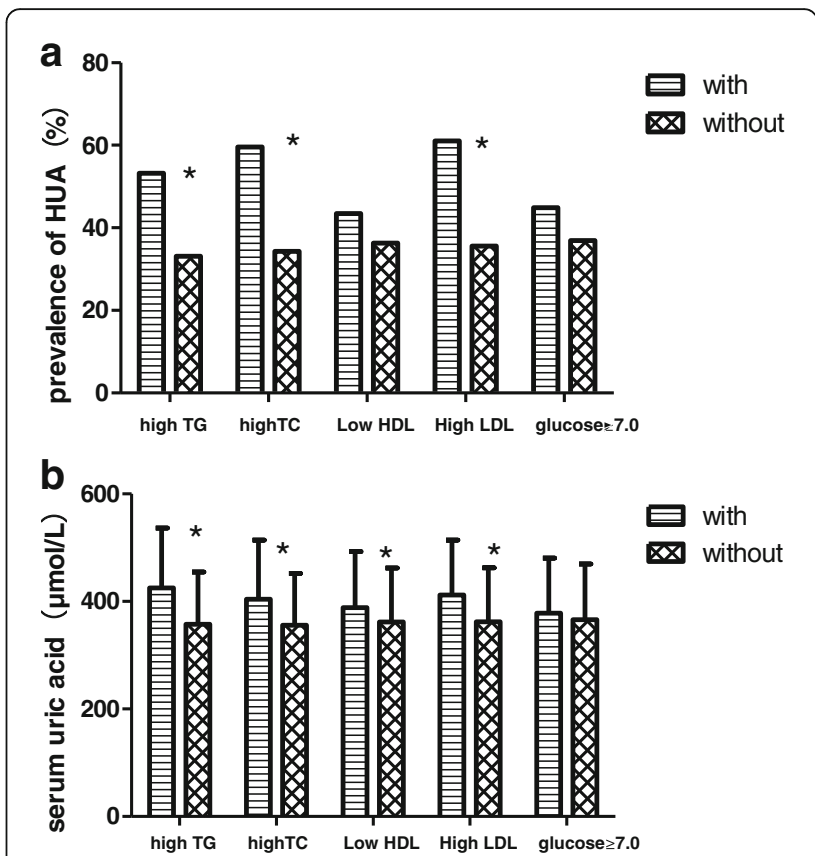

Fig. 2 Serum uric acid level and prevalence of hyperuricemia with or without selected risk factors. a prevalence of hyperuricemia, $\mathbf{b}$ Serum uric acid level. \#P $<0.05 ; * P<0.001$

associated with lower concentrations of uric acid in a small group [31].

Low to moderate education level was also a risk factor of hyperuricemia in our study. Some researchers have found that well-educated individuals appeared to have a healthier and more balanced diet [32-34]. This may be because people who have low to moderate levels of education have fewer opportunities to accumulate nutritional knowledge and may, therefore, pay less attention to their dietary intake.

We also found being Tibetan rather than Han, and having a high level of physical activity were protective factors for hyperuricemia in men. A review found that the risk of developing hyperuricemia and gout varied across populations by race and ethnicity [35]. Tibetans have lived on the plateau for generations isolated from inland China and have different genetic backgrounds, with a lower level of heterozygosity, and a higher level of runs of homozygosity [36]. This could explain some at least of the ethnic differences in our study, although there is no current direct evidence of a genetic link. The relationship between physical activity and uric acid or hyperuricemia is not entirely clear. Vigorous intensity activity has been shown to increase circulating uric acid level [37]. People at high altitude, however, tend to take only mild to moderate intensity activity because of the hypoxic environment. Yamanaka et al. stated that muscle exercise, at a degree below the anaerobic threshold, did not cause major purine nucleotide degradation and may 
Table 6 Characteristics of subjects with and without hyperuricemia according to gender

\begin{tabular}{|c|c|c|c|c|c|c|}
\hline & \multicolumn{3}{|c|}{ Male $(\mathrm{N}=614)$} & \multicolumn{3}{|c|}{ Female $(\mathrm{N}=802)$} \\
\hline & $\begin{array}{l}\mathrm{HAU} \\
(N=250)\end{array}$ & $\begin{array}{l}\text { without HAU } \\
(N=364)\end{array}$ & OR $(95 \% \mathrm{Cl})$ & $\begin{array}{l}\mathrm{HAU} \\
(N=276)\end{array}$ & $\begin{array}{l}\text { without HUA } \\
(N=526)\end{array}$ & OR $(95 \% \mathrm{Cl})$ \\
\hline High TG & $20.80 \%$ & $8.7 \%^{*}$ & $2.13(1.156-3.926)^{\#}$ & $18.10 \%$ & $7.2 \%{ }^{*}$ & $1.549(0.887-2.704)$ \\
\hline High TC & $32 \%$ & $13.2 \%^{*}$ & $2.313(1.364-3.923)^{*}$ & $27.90 \%$ & $17.3 \%^{*}$ & $1.088(0.699-1.694)$ \\
\hline Low HDL & $20.10 \%$ & $21.80 \%$ & $1.334(0.791-2.249)$ & $14.10 \%$ & $7.3 \%{ }^{\#}$ & $1.706(0.925-3.145)$ \\
\hline High LDL & $10.00 \%$ & $4.50 \%$ & $1.896(0.866-4.15)$ & $13.00 \%$ & $4.4 \%^{*}$ & $2.696(1.386-5.245)^{*}$ \\
\hline Glucose $\geq 7.0$ & $5.40 \%$ & $5.40 \%$ & $0.559(0.217-1.439)$ & $8.60 \%$ & $4.90 \%$ & $1.039(0.463-2.333)$ \\
\hline
\end{tabular}

Adjusted for age, BMI, WHR, serum creatinine, current smoking, current drinking, ethnicity, education, physical activity, occupation, eating meat and drinking buttered tea

$B M I$ body mass index, HDL-C high-density lipoprotein cholesterol, $L D L-C$ low-density lipoprotein cholesterol, high TC hypercholesterolemia, high $T G$ hypertriglyceridemia, High TC TC $\geq 6.21 \mathrm{mmol} / \mathrm{L}$, low HDL-C HDL-C $<1.03 \mathrm{mmol} / \mathrm{L}$, high LDL-C LDL-C $\geq 4.16 \mathrm{mmol} / \mathrm{L}, H i g h$ TG TG $\geq 2.26 \mathrm{mmol} / \mathrm{L} .{ }^{*} P<0.001 ;{ }^{*} P<0.05$

be beneficial for people with gout or hyperuricemia [38]. Another study found that moderate intensity physical activity was associated with the lower uric acid concentrations in obese individuals [39].

A lot of epidemiologic studies have reported a relationship between serum uric acid levels and a number of cardiovascular conditions, including hypertension, metabolic syndrome, coronary artery disease, cerebrovascular disease, vascular dementia, preeclampsia and kidney disease [40]. We found that serum uric acid was positively related to TG and TC and negatively related to HDL-c in all subjects, and that hyperuricemia was a risk factor for high TG and high TC in men and high LDL-c in women. Research in cell cultures and animal models have explored possible mechanisms that might explain the relationship between hyperuricemia and dyslipidemia. The first possible mechanism is inflammatory and oxidative reaction induced by uric acid in adipocytes, which was important in causing metabolic syndrome in obese mice [41].The second possible mechanism is that overexpressed xanthine oxidoreductase in adipocytes is critical to the process of adipogenesis.

\section{Limitations}

This study has several limitations. First, it was a crosssectional study, so could not provide any information about cause and effect. Prospective studies are required for further investigation of these findings. Second, the prevalence of hyperuricemia was assessed by a single test of blood samples, which may generate errors. Thirdly, this study was conducted only in Ganzi Tibetan Autonomous Prefecture, and so may not reflect the prevalence of hyperuricemia across the wider Tibetan area. More epidemiological studies are required to investigate the prevalence of hyperuricemia and its risk factors in high altitude areas and across Tibet. The doctors and nurses in this study were trained in how to obtain research data using a standardized measurement protocol, but measurements from a single visit might lead to incorrect values for the anthropometric indexes and language difference might result in incorrect data for medical history and lifestyle. Finally, we did not evaluate dietary structure by using a food frequency questionnaire, which is in an important component in the prevalence of hyperuricemia.

\section{Conclusion}

In conclusion, the prevalence of hyperuricemia is relatively high in Ganzi Tibetan Autonomous Prefecture, China. Being farmers-herdsmen, having low to moderate education level, current drinking, hypertension, and having higher BMI (1 unit increase) and creatinine (1 unit increase) all contributed to hyperuricemia in this population. It is crucial for the government to pay more attention to preventing and managing hyperuricemia in this area.

\section{Abbreviations}

BMI: Body mass index; DBP: Diastolic blood pressure; FPG: Fasting plasma glucose; HC: Hip circumference; HDL-C: High-density lipoprotein cholesterol; HUA: Hyperuricemia; LDL-C: Low-density lipoprotein cholesterol; SBP: Systolic blood pressure; TC: Total cholesterol; TG: Triglycerides; WC: Waist

circumference; WHR: Waist-to-hip ratio

\section{Acknowledgements}

The authors thank the teams led by Dr.R Renqian Lachu and Dr.R Hongfen Zhu at Jiulong County People's Hospital, Tibetan Autonomous Prefecture, People's Republic of China for their contributions to this study. We thank Melissa Leffler, MBA, from Liwen Bianji, Edanz Editing China (www.liwenbianji.cn/ac), for editing the English text of a draft of this manuscript.

\section{Funding}

This study was funded by two Science and Technology Pillar Programs in Sichuan Province (Grant no.2012SZ0131 and 2017SZ0008). The funding body had no role in the design of the study, collection, analysis and interpretation of the data, or writing the manuscript.

\section{Availability of data and materials}

The datasets used and/or analyzed during the current study are available from the corresponding author upon reasonable request.

\section{Authors' contributions}

XZ, QTM and XPC designed the study and participated in collecting data. XZ, JYF and HL undertook the statistical analysis and wrote this manuscript. RFS and DS collected data and blood sample. LCRQ, ZELT and YBD recruited participants in Tibetan area Sichuan province. XPC revised the manuscript for important intellectual content and languages. All authors read and approved the final manuscript. 


\section{Ethics approval and consent to participate}

The research protocol was reviewed by the Medical Ethics committee of West China Hospital, Sichuan University. The study protocol conforms with the ethical guidelines of the Declaration of Helsinki. Informed written consent was obtained from patients before enrollment. The Medical Ethics Committee of West China Hospital Sichuan University approved all procedures.

\section{Consent for publication}

All co-authors and participants have given their consent for publication of this article in Lipids in Health and Disease.

\section{Competing interests}

The authors declare that they have no competing interests (financial, political, personal, religious, ideological, academic, intellectual, or any other) to declare in relation to this manuscript.

\section{Publisher's Note}

Springer Nature remains neutral with regard to jurisdictional claims in published maps and institutional affiliations.

\section{Author details}

'Department of Cardiology, West China Hospital, Sichuan University, Chengdu, Sichuan 610000, People's Republic of China. ${ }^{2}$ Jiulong County People's Hospital, Jiulong 616200, Tibetan Autonomous Prefecture, People's Republic of China. ${ }^{3} G a n z i$ Tibetan Autonomous Prefecture People's Hospital, Kangding 626000, Tibetan Autonomous Prefecture, People's Republic of China.

Received: 2 July 2018 Accepted: 1 October 2018

\section{Published online: 12 October 2018}

\section{References}

1. Multidisciplinary Expert Task Force on H, Related D. Chinese multidisciplinary expert consensus on the diagnosis and treatment of hyperuricemia and related diseases. Chin Med J. 2017;130(20):2473-88

2. Yu KH, Chen DY, Chen JH, Chen SY, Chen SM, Cheng TT, Hsieh SC, Hsieh TY, Hsu PF, Kuo CF, et al. Management of gout and hyperuricemia: multidisciplinary consensus in Taiwan. Int J Rheum Dis. 2018;21(4):772-87.

3. Zhu Y, Pandya BJ, Choi HK. Prevalence of gout and hyperuricemia in the US general population: the National Health and nutrition examination survey 2007-2008. Arthritis Rheum. 2011;63(10):3136-41.

4. Liu H, Zhang XM, Wang YL, Liu BC. Prevalence of hyperuricemia among Chinese adults: a national cross-sectional survey using multistage, stratified sampling. J Nephrol. 2014:27(6):653-8.

5. Liu R, Han C, Wu D, Xia X, Gu J, Guan H, Shan Z, Teng W. Prevalence of hyperuricemia and gout in mainland China from 2000 to 2014: a systematic review and meta-analysis. Biomed Res Int. 2015;2015:762820.

6. Huang X, Zhou Z, Liu J, Song W, Chen Y, Liu Y, Zhang M, Dai W, Yi Y, Zhao S. Prevalence, awareness, treatment, and control of hypertension among China's Sichuan Tibetan population: a cross-sectional study. Clin Exp Hypertens. 2016;38(5):457-63.

7. Liu L, Liu L, Ding Y, Huang Z, He B, Sun S, Zhao G, Zhang H, Miki T, Mizushima $\mathrm{S}$, et al. Ethnic and environmental differences in various markers of dietary intake and blood pressure among Chinese Han and three other minority peoples of China: results from the WHO cardiovascular diseases and alimentary comparison (CARDIAC) study. Hypertens Res. 2001;24(3):315-22.

8. Xu S, Jiayong Z, Li B, Zhu H, Chang H, Shi W, Gao Z, Ning X, Wang J. Prevalence and clustering of cardiovascular disease risk factors among Tibetan adults in China: a population-based study. PLoS One. 2015;10(6): e0129966

9. Sherpa LY, Deji, Stigum H, Chongsuvivatwong V, Nafstad P, Bjertness E. Prevalence of metabolic syndrome and common metabolic components in high altitude farmers and herdsmen at $3700 \mathrm{~m}$ in Tibet. High Alt Med Biol. 2013;14(1):37-44

10. Xu S, Wang Q, Liu J, Bian B, Yu X, Yu X, Ning X, Wang J. The prevalence of and risk factors for diabetes mellitus and impaired glucose tolerance among Tibetans in China: a cross-sectional study. Oncotarget. 2017;8(68):112467-76.

11. Shi R, Li J, He J, Meng Q, Qian Z, Shi D, Liu Q, Cai Y, Li X, Chen X. Association of with-no-lysine kinase 1 and serine/threonine kinase 39 gene polymorphisms and haplotypes with essential hypertension in Tibetans. Environ Mol Mutagen. 2018;59(2):151-60.
12. Liu K, He S, Hong B, Yang R, Zhou X, Feng J, Wang S, Chen X. Over time, do anthropometric measures still predict diabetes incidence in chinese han nationality population from Chengdu community? Int J Endocrinol. 2013; 2013:239376.

13. Li DD, Zou MN, Hu X, Zhang M, Jia CY, Tao CM, Wang LL, Ying BW. Reference intervals and factors contributing to serum cystatin C levels in a Chinese population. J Clin Lab Anal. 2012;26(2):49-54.

14. Expert Panel on Detection $E$, Treatment of High Blood Cholesterol in A. Executive summary of the third report of the National Cholesterol Education Program (NCEP) expert panel on detection, evaluation, and treatment of high blood cholesterol in adults (adult treatment panel III). Jama. 2001;285(19):2486-97.

15. Wang CN, Liang Z, Wei P, Liu P, Yu JX, Zhang DM, Ma FL. Changes in dietary patterns and certain nutrition-related diseases in urban and rural residents of Jiangsu Province, China, during the 1990s. Biomed Environ Sci. 2002;15(4):271-6.

16. Conen D, Wietlisbach V, Bovet P, Shamlaye C, Riesen W, Paccaud F, Burnier M. Prevalence of hyperuricemia and relation of serum uric acid with cardiovascular risk factors in a developing country. BMC Public Health. 2004:4:9.

17. Cuspidi C, Valerio C, Sala C, Meani S, Esposito A, Zanchetti A, Mancia G. Lack of association between serum uric acid and organ damage in a nevertreated essential hypertensive population at low prevalence of hyperuricemia. Am J Hypertens. 2007;20(6):678-85.

18. Sofi F, Dinu M, Pagliai G, Cesari F, Marcucci R, Casini A. Mediterranean versus vegetarian diet for cardiovascular disease prevention (the CARDIVEG study): study protocol for a randomized controlled trial. Trials. 2016;17(1):233.

19. Kimura Y, Okumiya K, Sakamoto R, Ishine M, Wada T, Kosaka Y, Wada C, Ishimoto $Y$, Hirosaki M, Kasahara $Y$, et al. Comprehensive geriatric assessment of elderly highlanders in Qinghai, China IV: comparison of food diversity and its relation to health of Han and Tibetan elderly. Geriatr Gerontol Int. 2009;9(4):359-65.

20. Ge K, Zhai F, Wang Q. Effect of nationality on dietary pattern and meal behavior in China. Am J Clin Nutr. 1997;65(4 Suppl):1290S-4S.

21. Torralba KD, De Jesus E, Rachabattula S. The interplay between diet, urate transporters and the risk for gout and hyperuricemia: current and future directions. Int J Rheum Dis. 2012;15(6):499-506.

22. Snaith M. Gout and alcohol. Rheumatology. 2004;43(10):1208-9.

23. Chuang SY, Lee SC, Hsieh YT, Pan WH. Trends in hyperuricemia and gout prevalence: nutrition and health survey in Taiwan from 1993-1996 to 20052008. Asia Pac J Clin Nutr. 2011;20(2):301-8.

24. Riches PL, Wright AF, Ralston $\mathrm{SH}$. Recent insights into the pathogenesis of hyperuricaemia and gout. Hum Mol Genet. 2009;18(R2):R177-84.

25. Faller J, Fox $\mid \mathrm{H}$. Ethanol-induced hyperuricemia: evidence for increased urate production by activation of adenine nucleotide turnover. N Engl J Med. 1982:307(26):1598-602

26. Nakamura K, Sakurai M, Miura K, Morikawa Y, Yoshita K, Ishizaki M, Kido T, Naruse $Y$, Suwazono $Y$, Nakagawa $\mathrm{H}$. Alcohol intake and the risk of hyperuricaemia: a 6-year prospective study in Japanese men. Nutr Metabol Cardiovasc Dis. 2012;22(11):989-96.

27. Choi HK, Atkinson K, Karlson EW, Curhan G. Obesity, weight change, hypertension, diuretic use, and risk of gout in men: the health professionals follow-up study. Arch Intern Med. 2005;165(7):742-8.

28. Kaufmann $H$, Freeman R, Biaggioni I, Low P, Pedder S, Hewitt LA, Mauney J, Feirtag M, Mathias CJ, Investigators NOH. Droxidopa for neurogenic orthostatic hypotension: a randomized, placebo-controlled, phase 3 trial. Neurology. 2014;83(4):328-35.

29. Yang J, Liu Z, Zhang C, Zhao Y, Sun S, Wang S, Zhao Y, Zhang Y, Li J, Lu F. The prevalence of hyperuricemia and its correlates in an inland Chinese adult population, urban and rural of Jinan. Rheumatol Int. 2013;33(6):1511-7.

30. Tsai YT, Liu JP, Tu YK, Lee MS, Chen PR, Hsu HC, Chen MF, Chien KL. Relationship between dietary patterns and serum uric acid concentrations among ethnic Chinese adults in Taiwan. Asia Pac J Clin Nutr. 2012;21(2):263-70.

31. Szeto YT, Kwok TC, Benzie IF. Effects of a long-term vegetarian diet on biomarkers of antioxidant status and cardiovascular disease risk. Nutrition. 2004;20(10):863-6.

32. Johansson L, Thelle DS, Solvoll K, Bjorneboe GE, Drevon CA. Healthy dietary habits in relation to social determinants and lifestyle factors. Br J Nutr. 1999; 81(3):211-20.

33. Hulshof KF, Lowik MR, Kok FJ, Wedel M, Brants HA, Hermus RJ, ten Hoor F. Diet and other life-style factors in high and low socio-economic groups (Dutch nutrition surveillance system). Eur J Clin Nutr. 1991;45(9):441-50. 
34. Wang Z, Dang S, Yan H. Nutrient intakes of rural Tibetan mothers: a crosssectional survey. BMC Public Health. 2010;10:801.

35. MacFarlane LA, Kim SC. Gout: a review of nonmodifiable and modifiable risk factors. Rheum Dis Clin N Am. 2014;40(4):581-604.

36. Lu D, Lou H, Yuan K, Wang X, Wang Y, Zhang C, Lu Y, Yang X, Deng L, Zhou $Y$, et al. Ancestral origins and genetic history of Tibetan highlanders. Am J Hum Genet. 2016;99(3):580-94.

37. Sutton JR, Toews $\mathrm{CJ}$, Ward GR, Fox IH. Purine metabolism during strenuous muscular exercise in man. Metab Clin Exp. 1980;29(3):254-60.

38. Yamanaka H, Kawagoe Y, Taniguchi A, Kaneko N, Kimata S, Hosoda S, Kamatani N, Kashiwazaki S. Accelerated purine nucleotide degradation by anaerobic but not by aerobic ergometer muscle exercise. Metab Clin Exp. 1992:41(4):364-9.

39. Nishida Y, lyadomi M, Higaki Y, Tanaka H, Hara M, Tanaka K. Influence of physical activity intensity and aerobic fitness on the anthropometric index and serum uric acid concentration in people with obesity. Intern Med. 2011; 50(19):2121-8

40. Feig DI, Kang DH, Johnson RJ. Uric acid and cardiovascular risk. N Engl J Med. 2008;359(17):1811-21.

41. Furukawa S, Fujita T, Shimabukuro M, Iwaki M, Yamada Y, Nakajima Y, Nakayama O, Makishima M, Matsuda M, Shimomura I. Increased oxidative stress in obesity and its impact on metabolic syndrome. J Clin Invest. 2004; 114(12):1752-61.

Ready to submit your research? Choose BMC and benefit from:

- fast, convenient online submission

- thorough peer review by experienced researchers in your field

- rapid publication on acceptance

- support for research data, including large and complex data types

- gold Open Access which fosters wider collaboration and increased citations

- maximum visibility for your research: over $100 \mathrm{M}$ website views per year

At $\mathrm{BMC}$, research is always in progress.

Learn more biomedcentral.com/submissions 